S3 Table. TPP PvB1: Point-of-care diagnosis of subpatent Plasmodium vivax infection

\begin{tabular}{|c|c|c|c|c|}
\hline Type & Characteristic & Minimal (M) / Description & Optimal (O) & Comment \\
\hline \multirow[t]{5}{*}{ Scope } & Intended use & \multicolumn{3}{|c|}{$\begin{array}{l}\text { The test goal is to provide an infection detection test for } P \text {. vivax able to detect a substantial fraction of all infections } \\
\text { (symptomatic or asymtptomatic) for test-and-treat in any active infection detection interventions. Therefore, the test } \\
\text { needs to accurately detect low density erythrocytic forms of } P \text {. vivax in a point-of-care manner. }\end{array}$} \\
\hline & Test outcome & \multicolumn{3}{|c|}{ Guide blood-stage and, if appropriate, liver-stage treatment in reactive and proactive case detection } \\
\hline & Target population & \multicolumn{3}{|c|}{$\begin{array}{l}\text { The target population is any individual susceptible to suffer from a latent infection from } P . \text { vivax, including neonates, } \\
\text { children, and pregnant women to enable treatment and, if appropriate, radical cure. }\end{array}$} \\
\hline & Target users & \multicolumn{3}{|c|}{$\begin{array}{l}\text { The target users include community health workers with minimal training and any health worker with a similar or } \\
\text { superior training level. }\end{array}$} \\
\hline & Implementation level & \multicolumn{3}{|c|}{$\begin{array}{l}\text { The target implementation levels are community health facilities, health posts, health centers, district hospital as well as } \\
\text { reference centers [5]. }\end{array}$} \\
\hline \multirow[t]{4}{*}{ Performance } & Analytical sensitivity & $\begin{array}{l}\text { Limit of detection for target } \\
\text { analyte corresponding to a } \\
\text { peripheral parasitaemia of } 20 \\
\mathrm{p} / \mu \mathrm{L}\end{array}$ & $\begin{array}{l}\text { Limit of detection for target } \\
\text { analyte corresponding to a } \\
\text { peripheral parasitaemia of } 1 \\
\text { p/ } \mu \mathrm{L}\end{array}$ & $\begin{array}{l}\text { "M" corresponds to a ten-fold increase in } \\
\text { sensitivity as compared to RDTs and is line with } \\
\text { malERA recommendation. "O" corresponds to a } \\
\text { hundred-fold increase in sensitivity as compared } \\
\text { to RDTs. For indirect tests (e.g. serology), the } \\
\text { analytical sensitivity might not directly relate to } \\
\text { parasitaemia. }\end{array}$ \\
\hline & Analytical specificity & $\begin{array}{l}\text { Discriminate between } P \text {. vivax } \\
\text { and other Plasmodium spp. Do } \\
\text { not cross-react with any other } \\
\text { pathogen infecting humans }\end{array}$ & $\begin{array}{l}\text { Discriminate between } P \text {. vivax, } \\
P . \text { falciparum and other } \\
\text { Plasmodium spp. Do not cross- } \\
\text { react with any other pathogen } \\
\text { infecting humans }\end{array}$ & $\begin{array}{l}\text { "M" enables the specific identification of } P \text {. } \\
\text { vivax. "O" provides a unique test for } P \text {. vivax and } \\
P \text {. falciparum co-endemicity areas. Cross- } \\
\text { reactivity between } P \text {. vivax and } P \text {. ovale might be } \\
\text { beneficial to identify both of these relapsing } \\
\text { species. }\end{array}$ \\
\hline & Diagnostic outcome & Binary & Binary & $\begin{array}{l}\text { A continuous (quantitative) outcome is not } \\
\text { required for the intended use of the test. }\end{array}$ \\
\hline & Diagnostic sensitivity & $\begin{array}{l}>95 \% \text { as compared to standard } \\
\text { PCR with known limit of } \\
\text { detection of } 1 \mathrm{p} / \mu \mathrm{L}\end{array}$ & $\begin{array}{l}\geq 99 \% \text { as compared to standard } \\
\text { PCR with known limit of } \\
\text { detection of } 1 \mathrm{p} / \mu \mathrm{L}\end{array}$ & $\begin{array}{l}\text { In line with malERA recommendations (but } \\
\text { comparator not specified) [1]. Comparator might } \\
\text { need to be adapted for indirect tests (e.g. based } \\
\text { on serology). }\end{array}$ \\
\hline
\end{tabular}




\begin{tabular}{|c|c|c|c|c|}
\hline Type & Characteristic & Minimal (M) / Description & Optimal (O) & Comment \\
\hline & Diagnostic specificity & $\begin{array}{l}>95 \% \text { as compared to standard } \\
\text { PCR with known limit of } \\
\text { detection of } 1 \mathrm{p} / \mu \mathrm{L}\end{array}$ & $\begin{array}{l}\geq 99 \% \text { as compared to standard } \\
\text { PCR with known limit of } \\
\text { detection of } 1 \mathrm{p} / \mu \mathrm{L}\end{array}$ & \\
\hline & $\begin{array}{l}\text { Repeatability (inter- } \\
\text { operators) }\end{array}$ & Kappa $>0.8$ & Kappa $>0.9$ & $\begin{array}{l}\text { Kappa statistic can be used to evaluate binary } \\
\text { outcomes agreement. Suggested values are } \\
\text { arbitrary. }\end{array}$ \\
\hline & $\begin{array}{l}\text { Reproducibility (inter- } \\
\text { laboratories) }\end{array}$ & Kappa $>0.7$ & Kappa $>0.9$ & See Repeatability \\
\hline \multirow[t]{8}{*}{$\begin{array}{l}\text { Operational } \\
\text { aspects }\end{array}$} & Assay format & $\begin{array}{l}\text { End point, single-use in vitro } \\
\text { diagnostic }\end{array}$ & $\begin{array}{l}\text { End point single-use in vitro } \\
\text { diagnostic }\end{array}$ & $\begin{array}{l}\text { The assay format should enable the individual } \\
\text { testing of } 100 \text { or less individuals in a point-of- } \\
\text { care manner. }\end{array}$ \\
\hline & Assay throughput & Single assessment per test & $\begin{array}{l}\text { Single assessment per test, } \\
\text { optional batch testing for higher } \\
\text { throughput }\end{array}$ & See assay format \\
\hline & Assay packaging & $\begin{array}{l}\text { Package of single kits sharing } \\
\text { reagents (if required) and user } \\
\text { manual }\end{array}$ & $\begin{array}{l}\text { Package of single kits with } \\
\text { individual reagents sharing user } \\
\text { manual }\end{array}$ & $\begin{array}{l}\text { "M" and "O" reflect current packaging formats of } \\
\text { RDTs. }\end{array}$ \\
\hline & Operation conditions & $\begin{array}{l}5^{\circ} \mathrm{C}-40^{\circ} \mathrm{C} \\
\text { Up to } 90 \% \text { relative humidity } \\
(\mathrm{RH})\end{array}$ & $\begin{array}{l}5^{\circ} \mathrm{C}-45^{\circ} \mathrm{C} \\
\text { Up to } 90 \% \mathrm{RH}\end{array}$ & $\begin{array}{l}\text { "M" and "O" reflect extreme conditions of } \\
\text { endemic countries. RDT transportation and } \\
\text { storage temperatures regularly exceed } 30^{\circ} \mathrm{C} \text {, } \\
\text { rarely } 40^{\circ} \mathrm{C}[10] .\end{array}$ \\
\hline & $\begin{array}{l}\text { Transportation and storage } \\
\text { stability }\end{array}$ & $\begin{array}{l}\geq 12 \text { months at } 35^{\circ} \mathrm{C} \text { and } 70 \% \\
\mathrm{RH} \text { with transport stress ( } 3 \text { days } \\
\left.\text { at } 60{ }^{\circ} \mathrm{C}\right) \text {, no cold chain needed }\end{array}$ & $\begin{array}{l}\geq 12 \text { months at } 45^{\circ} \mathrm{C} \text { and } 90 \% \\
\text { RH with transport stress ( } 3 \text { days } \\
\text { at } 60{ }^{\circ} \mathrm{C} \text { ), no cold chain needed }\end{array}$ & $\begin{array}{l}\text { "M" and "O" reflect typical and possible extreme } \\
\text { transportation and storage conditions observed } \\
\text { for RDTs [10]. }\end{array}$ \\
\hline & In use stability & $\begin{array}{l}>30 \text { minutes for single-use test } \\
\text { once opened }\end{array}$ & $\begin{array}{l}>2 \text { hours for single-use test } \\
\text { once opened }\end{array}$ & $\begin{array}{l}\text { For batch testing, this characteristics is likely to } \\
\text { impact the assay throughput. }\end{array}$ \\
\hline & Reagents reconstitution & $\begin{array}{l}\text { Reconstitution of reagent } \\
\text { acceptable if number of step is } \\
\text { limited }(\leq 5) \text { and not requiring } \\
\text { external equipment }\end{array}$ & $\begin{array}{l}\text { All reagents provided and ready } \\
\text { to use. }\end{array}$ & $\begin{array}{l}\text { "M" is more stringent than the actual } \\
\text { characteristic of LM (Giemsa solution } \\
\text { preparation requires several precise steps). "O" is } \\
\text { met by current RDTs. }\end{array}$ \\
\hline & Equipment & $\begin{array}{l}\text { Small }\left(\leq 100 \mathrm{~cm}^{2} \text { footprint }\right) \text { and } \\
\text { portable }(\leq 5 \mathrm{~kg})\end{array}$ & None & \\
\hline
\end{tabular}

Page 2 of 6 


\begin{tabular}{|c|c|c|c|c|}
\hline Type & Characteristic & Minimal (M) / Description & Optimal (O) & Comment \\
\hline & Power requirement & $\begin{array}{l}\text { Battery operated with } \geq 24 \\
\text { hours testing autonomy }\end{array}$ & None & \\
\hline & Maintenance & $\leq$ once per year & None & \\
\hline & Sample type & Capillary blood & $\begin{array}{l}\text { Capillary blood or any less } \\
\text { invasive validated sample }\end{array}$ & $\begin{array}{l}\text { Sample types less invasive than capillary blood } \\
\text { include saliva, urine, breath or transdermal } \\
\text { detection [11]. }\end{array}$ \\
\hline & Sample volume & $\leq 100 \mu \mathrm{L}$ of capillary blood & $\leq 50 \mu \mathrm{L}$ of capillary blood & Variable for other sample types \\
\hline & Sample preparation & $\leq 5$ steps & None & $\begin{array}{l}\text { "M" reflects the actual characteristic of LM (i.e. } \\
\text { fix, rinse, stain, rinse, dry). "O" is met by current } \\
\text { RDTs. }\end{array}$ \\
\hline & Overall test preparation & $\begin{array}{l}\leq 10 \text { steps, of which } \leq 2 \text { are } \\
\text { timed }\end{array}$ & $\leq 3$ steps, of which $\leq 1$ is timed & $\begin{array}{l}\text { "M" and "O" reflect actual characteristics of LM } \\
\text { and RDT. }\end{array}$ \\
\hline & Time-to-result & $\leq 30$ minutes & $\leq 10$ minutes & $\begin{array}{l}\text { Point-of-care testing requires short time-to-result } \\
\text { values. }\end{array}$ \\
\hline & Internal control & Included & Included & \\
\hline & External control & Available & Included & $\begin{array}{l}\text { External controls, such as positive control wells } \\
\text { for RDT, are especially important in low endemic } \\
\text { settings (i.e. in area of low positivity rate). }\end{array}$ \\
\hline & Assay interpretation & $\begin{array}{l}\text { Unequivocal, recorded by } \\
\text { operator }\end{array}$ & $\begin{array}{l}\text { Unequivocal, recorded by } \\
\text { operator or electronically }\end{array}$ & \\
\hline & Data capture & Manual by operator & Electronic automated & \\
\hline & Data transfer & Manual by operator & $\begin{array}{l}\text { Automated via internet or GSM } \\
\text { connectivity }\end{array}$ & \\
\hline & Training & $\begin{array}{l}\leq 5 \text { days for inexperienced } \\
\text { health worker }\end{array}$ & $\begin{array}{l}\leq 1 \text { day for inexperienced health } \\
\text { worker }\end{array}$ & $\begin{array}{l}\text { Include plan for quality control and proficiency } \\
\text { monitoring. }\end{array}$ \\
\hline & Biosafety & $\begin{array}{l}\text { Class B IVD (moderate } \\
\text { individual and low public health } \\
\text { risk) }\end{array}$ & $\begin{array}{l}\text { Class A IVD (low individual } \\
\text { and public health risk) }\end{array}$ & $\begin{array}{l}\text { According to risk-based classification of } \\
\text { diagnostics for WHO prequalification [13]. }\end{array}$ \\
\hline
\end{tabular}




\begin{tabular}{|c|c|c|c|c|}
\hline Type & Characteristic & Minimal (M) / Description & Optimal (O) & Comment \\
\hline & Language & $\begin{array}{l}\text { English, Spanish and } \\
\text { Portuguese }\end{array}$ & Local languages & \\
\hline \multirow[t]{2}{*}{ Cost } & End user price per test & $\leq 2.0 \mathrm{USD}$ & $\leq 1.0 \mathrm{USD}$ & $\begin{array}{l}\text { "O" is more stringent than malERA } \\
\text { recommendation [1]. }\end{array}$ \\
\hline & Cost of diagnosis & $\leq 5.0 \mathrm{USD}$ & $\leq 2.0 \mathrm{USD}$ & $\begin{array}{l}\text { RDT and LM costs of diagnosis were reported to } \\
\text { be between } 2.0 \text { and 1.0 USD in } 2011 \text { in Uganda } \\
{[14] \text {. }}\end{array}$ \\
\hline
\end{tabular}




\section{Supplementary References}

1. The malERA Consultative Group on Diagnoses and Diagnostics. A Research Agenda for Malaria Eradication: Diagnoses and Diagnostics. PLoS Med. 2011;8: e1000396. doi:10.1371/journal.pmed.1000396.t001

2. World Health Organization. WHO Evidence Review Group on Malaria Diagnosis in Low Transmission Settings [Internet]. 21 Mar 2014 [cited 11 Oct 2015] pp. 1-33. Available: http://www.who.int/malaria/mpac/mpac_mar2014_diagnosis_low_transmission_setting s_report.pdf

3. PATH. Target Product Profile: Point-of-Care Malaria Infection Detection Test. In: sites.path.org [Internet]. [cited 25 Jun 2016]. Available: http://sites.path.org/dx/files/2012/11/DIAMETER_IDT_TPP_FINAL_forwebsite.pdf

4. World Health Organization. Control and Elimination of Plasmodium Vivax Malaria. 2015.

5. FIND. Strategy for Malaria 2015-2020 [Internet]. 16 Sep 2015 [cited 6 Oct 2015] pp. 1-24. Available: http://www.finddiagnostics.org/export/sites/default/resourcecentre/find_reports/pdfs/FIND_malaria_strategy_web_v03-2015.pdf

6. McKenzie FE, Jeffery GM, Collins WE. Plasmodium vivax blood-stage dynamics. J Parasitol. 2002;88: 521-535. doi:10.1645/00223395(2002)088[0521:PVBSD]2.0.CO;2

7. McKenzie FE, Jeffery GM, Collins WE. Gametocytemia and fever in human malaria infections. J Parasitol. 2007;93: 627-633. doi:10.1645/GE-1052R.1

8. Barber BE, William T, Grigg MJ, Parameswaran U, Piera KA, Price RN, et al. Parasite Biomass-Related Inflammation, Endothelial Activation, Microvascular Dysfunction and Disease Severity in Vivax Malaria. Stevenson MM, editor. PLoS Pathog. Public Library of Science; 2015;11: e1004558. doi:10.1371/journal.ppat.1004558

9. Abba K, Kirkham AJ, Olliaro PL, Deeks JJ, Donegan S, Garner P, et al. Rapid diagnostic tests for diagnosing uncomplicated non-falciparum or Plasmodium vivax malaria in endemic countries. Abba K, editor. Cochrane Database Syst Rev. Chichester, UK: John Wiley \& Sons, Ltd; 2014;12: CD011431. doi:10.1002/14651858.CD011431

10. Albertini A, Lee E, Coulibaly SO, Sleshi M, Faye B, Mationg ML, et al. Malaria rapid diagnostic test transport and storage conditions in Burkina Faso, Senegal, Ethiopia and the Philippines. Malar J. BioMed Central Ltd; 2012;11: 406. doi:10.1186/1475-2875$11-406$

11. Lukianova-Hleb EY, Campbell KM, Constantinou PE, Braam J, Olson JS, Ware RE, et al. Hemozoin-generated vapor nanobubbles for transdermal reagent- and needle-free detection of malaria. Proc Natl Acad Sci USA. 2014;111: 900-905. doi:10.1073/pnas.1316253111 
12. World Health Organization. Guidelines for the Treatment of Malaria. 3rd ed. 2015 Apr pp. 1-318.

13. World Health Organization. Risk Based Classification of Diagnostics for WHO Prequalification. In: who.int [Internet]. 2014 [cited 7 Oct 2015]. Available: http://www.who.int/diagnostics_laboratory/evaluations/140513_who_risk_based_classi fication_of_ivds_for_pq_buffet.pdf?ua $=1$

14. Batwala V, Magnussen P, Hansen KS, Nuwaha F. Cost-effectiveness of malaria microscopy and rapid diagnostic tests versus presumptive diagnosis: implications for malaria control in Uganda. Malar J. BioMed Central Ltd; 2011;10: 372. doi:10.1186/1475-2875-10-372

15. Hofmann N, Mwingira F, Shekalaghe S, Robinson LJ, Mueller I, Felger I. UltraSensitive Detection of Plasmodium falciparum by Amplification of Multi-Copy Subtelomeric Targets. Seidlein von L, editor. PLoS Med. Public Library of Science; 2015;12: e1001788. doi:10.1371/journal.pmed.1001788

16. Murphy SC, Prentice JL, Williamson K, Wallis CK, Fang FC, Fried M, et al. Real-time quantitative reverse transcription PCR for monitoring of blood-stage Plasmodium falciparum infections in malaria human challenge trials. Am J Trop Med Hyg. American Society of Tropical Medicine and Hygiene; 2012;86: 383-394. doi:10.4269/ajtmh.2012.10-0658 